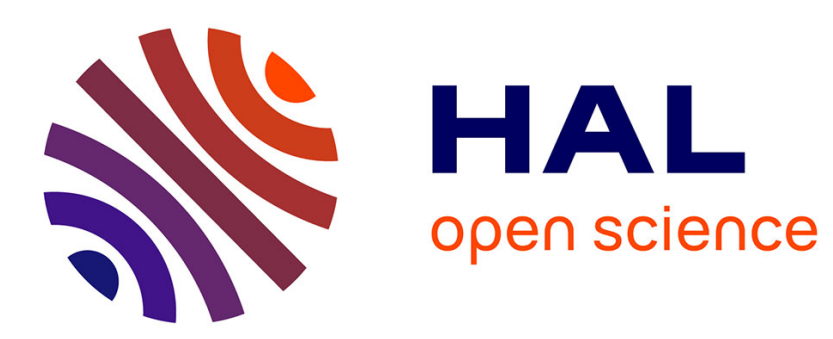

\title{
Subject-Specific Ligament Models: Towards Real-Time Simulation of the Knee Joint
}

Tobias Heimann, François Chung, Hans Lamecker, Hervé Delingette

\section{To cite this version:}

Tobias Heimann, François Chung, Hans Lamecker, Hervé Delingette. Subject-Specific Ligament Models: Towards Real-Time Simulation of the Knee Joint. Computational Biomechanics for Medicine IV Workshop 2009, 2009, London, United Kingdom. pp.107-119, 10.1007/978-1-4419-5874-7_12 . inria-00616129

\section{HAL Id: inria-00616129 \\ https://hal.inria.fr/inria-00616129}

Submitted on 8 Jul 2013

HAL is a multi-disciplinary open access archive for the deposit and dissemination of scientific research documents, whether they are published or not. The documents may come from teaching and research institutions in France or abroad, or from public or private research centers.
L'archive ouverte pluridisciplinaire HAL, est destinée au dépôt et à la diffusion de documents scientifiques de niveau recherche, publiés ou non, émanant des établissements d'enseignement et de recherche français ou étrangers, des laboratoires publics ou privés. 


\title{
Subject-Specific Ligament Models: Towards Real-Time Simulation of the Knee Joint
}

\author{
Tobias Heimann, François Chung, Hans Lamecker, and Hervé Delingette \\ Asclepios Project, INRIA Sophia Antipolis, France \\ tobias.heimann@inria.fr
}

\begin{abstract}
We present an efficient finite element method to simulate a transversely isotropic non-linear material for ligaments. The approach relies on tetrahedral elements and exploits the geometry to optimize computation of the derivatives of the strain energy. To better support incompressibilty, deviatoric and dilational responses are uncoupled and a penalty term controls volume preservation. We derive stress and elasticity tensors required for implicit solvers and verify our model against the FEBio software using a variety of load scenarios with synthetic shapes. The maximum node positioning error for ligament materials is $<5 \%$ for strains under physiological conditions.

To generate subject-specific ligament models, we propose a novel technique to estimate fiber orientation from segmented ligament geometry. The approach is based on an automatic centerline extraction and generation of the corresponding diffusion field. We present results for a medial collateral ligament segmented from standard MRI data. Results show the general viability of the method, but also the limitations of current MRI acquisitions. In the future, we hope to employ the presented techniques for real-time simulation of knee surgery.
\end{abstract}

\section{Introduction}

The human knee joint has been extensively studied in the field of biomechanics, both due to its complex anatomy and its high clinical relevance. It has to cope with high loads and large displacements during many daily activities. The knee ligaments, notably the two cruciate ligaments and the two collateral ligaments, play a key role in ensuring stability of the joint [1]. They are also at increased risk during high impact scenarios as occurring e.g. in many sports activities. In case of ligament rupture, kinematics of the knee are severely compromised, and sophisticated surgical interventions are required to restore its function. Virtual simulation of these surgeries for subject-specific cases combined with a subsequent analysis of resulting knee kinematics seem a promising way to increase success rates of interventions $[2,3]$.

The finite element method (FEM) has successfully been used to analyze a variety of problems regarding the knee joint [3-5]. To achieve realistic simulation of ligaments, sophisticated material models have been presented $[6,7]$. These models implement properties such as the transverse isotropy and non-linear stressstrain curve due to internal collagen fibers, and partly also viscoelastic behavior. 
In most works related to real-time simulation, however, material properties of ligaments are highly simplified to maintain the required frame-rate. Often, simple isotropic linear elasticity is used [8]. Picinbono et al. [9] presented an optimized FE model for tetrahedral elements, which also allowed transversely isotropic materials. However, the stress-strain relationship was still linear. A fast non-linear anisotropic material model was presented by Teran et al. [10], who use the finite volume method to simulate muscles and tendons. This approach is equivalent to FEM for constant strain linear basis functions. Recently, Joldes et al. [11] developed a highly efficient framework for computation of soft tissue deformation, which is based on Total Lagrangian and explicit time integration. This framework was also implemented on GPU and used for viscoelastic nonlinear soft tissue simulation by Taylor et al. [12].

In this work, we present a material model that incorporates the ligamentspecific properties of transverse isotropy and non-linear stress-strain curve and that is suitable for both explicit and implicit solvers. The model is aimed at real-time simulation and reaches high efficiency due to the use of linear tetrahedral elements, which leads to an optimization of a large part of the involved computations. We also address the problem of generating subject-specific models, which is neglected in many current publications: with transversely isotropic materials for ligaments, the main fiber orientation has to be known for each finite element in the simulation. Techniques as X-ray diffraction or optical diffraction that can be used for experiments with cadavers are not possible in vivo. To employ FEM simulations of the knee for surgery planning, fiber orientations have to be extracted from clinical imaging modalities as MRI or DT-MRI.

The remainder of this paper is organized as follows: in Sec. 2, we present our optimized FE model for ligaments. Subsequently, the method to determine local fiber orientation from segmented geometry is presented in Sec. 3. In Sec. 4, image data and rheological parameters to generate a subject-specific model of the medial collateral ligament (MCL) are described. In Sec. 5, we verify our FE model by comparing it to the established FEBio software ${ }^{1}$ and we present our model of the MCL. We close with a discussion of results and future work.

\section{Transversely isotropic hyperelasticity for tetrahedrons}

In this section, we present the strain energy for the employed material model and derive its stress tensor (first derivative) and elasticity tensor (second derivative). The latter one is required if the model is used for implicit or quasi-static analysis. Throughout this work, we follow the concepts presented by Delingette [13] for triangular finite elements. In particular, the elasticity tensor for each node is assembled by accumulating terms from all adjacent edges, which allows to exploit symmetry to reduce computations.

\footnotetext{
${ }^{1}$ FEBio is available at the website of the Musculoskeletal Research Laboratories of the University of Utah: http://mrl.sci.utah.edu/software.php
} 


\subsection{Properties of tetrahedral elements}

The tetrahedral element with linear basis function features a constant strain, which reduces integration to a multiplication by volume. Moreover, the deformation tensor can be calculated efficiently based on the current node positions $\mathbf{Q}_{i}$ and the shape vectors of the tetrahedron. Shape vectors $\mathbf{D}_{i}$ are defined by the rest configuration of the tetrahedron (with node positions $\mathbf{P}_{i}$ ) as:

$$
\mathbf{D}_{i}=\frac{s}{V_{0}}\left(\left(\mathbf{P}_{i \oplus 2}-\mathbf{P}_{i \oplus 1}\right) \wedge\left(\mathbf{P}_{i \oplus 3}-\mathbf{P}_{i \oplus 1}\right)\right)
$$

where $\wedge$ denotes the cross product, $V_{0}$ is the volume in rest configuration and $s=1$ for $i=2,4$, else $s=-1$. Shape vector $\mathbf{D}_{i}$ stands perpendicular to the face opposite node $i$ and points inward. For the many interesting properties of shape vectors, we refer the reader to [13]. Here, we note that the deformation gradient tensor $\nabla \boldsymbol{\Phi}$ can be expressed as:

$$
\nabla \boldsymbol{\Phi}=\sum_{i=1}^{4} \mathbf{Q}_{i} \otimes \mathbf{D}_{i}
$$

Consequently, the right Cauchy-Green deformation tensor $\mathbf{C}$ is:

$$
\mathbf{C}=\nabla \boldsymbol{\Phi}^{T} \nabla \boldsymbol{\Phi}=\sum_{i=1}^{4} \sum_{j=1}^{4}\left(\mathbf{Q}_{i} \cdot \mathbf{Q}_{j}\right)\left(\mathbf{D}_{i} \otimes \mathbf{D}_{j}\right)
$$

The Jacobian $J$ of the deformation gradient tensor $\nabla \boldsymbol{\Phi}$ can also be expressed as the ratio of volumes in deformed and in rest configuration: $J=V / V_{0}$. We sum up that the signed volume of a tetrahedron is given by:

$$
V=\frac{1}{6}\left(\left(\mathbf{Q}_{4}-\mathbf{Q}_{1}\right) \cdot\left(\left(\mathbf{Q}_{2}-\mathbf{Q}_{1}\right) \wedge\left(\mathbf{Q}_{3}-\mathbf{Q}_{1}\right)\right)\right)
$$

where the order of nodes is interchangably as long as the sign is kept.

As described in [13], forces on node $i$ can be determined directly by $\mathbf{F}_{i}=$ $-V \nabla \Phi \mathbf{S} \mathbf{D}_{i}$, where $\mathbf{S}$ is the second Piola-Kirchhoff tensor used for large deformations. Using the weak formulation for finite elements [14], the required derivative $\partial \mathbf{S} / \partial \mathbf{C}$ results in very complex expressions (see e.g. [6]). Therefore, we employ the Rayleigh-Ritz approach and derive the required expressions directly from the discretized strain energy, using individual node positions $\mathbf{Q}_{i}$.

\subsection{Strain energy}

We base our strain energy $W$ on the terms proposed by Weiss et al. [6] for incompressible, transversely isotropic materials. However, instead of using an Augmented Lagrangian method to ensure incompressibility, we employ a simple penalty approach based on the bulk modulus of the material. The energy is composed of three different terms:

$$
W=F_{G}\left(I_{1}\right)+F_{C}\left(I_{4}\right)+F_{V}(J)
$$


$F_{G}$ describes the contribution of the isotropic ground substance matrix, $F_{C}$ describes the contribution from the collagen fibers, and $F_{V}$ is the penalty term for volume changes. The underlying invariants are defined as:

$$
\begin{aligned}
I_{1} & =J^{-\frac{2}{3}} \operatorname{tr} \mathbf{C} \\
I_{4} & =J^{-\frac{2}{3}} \mathbf{a C a}=\lambda^{2} \\
J & =V / V_{0}
\end{aligned}
$$

Here, $I_{1}$ is a standard invariant of $\mathbf{C}$ for nearly incompressible materials [14]. $I_{4}$ arises from the anisotropy of the material, using main fiber orientation a [6]. $\lambda=l / l_{0}$ is the stretch along this direction. Note that in our formulation, $I_{1}$ and $I_{4}$ remain constant under a pure volume change. Thus, $F_{G}$ and $F_{C}$ represent a pure deviatoric response and $F_{V}$ a pure dilational response.

\subsection{Derivations for the Jacobian}

Starting with the formula for the volume of a tetrahedron (4), the first derivative of $J$ w.r.t. point $\mathbf{Q}_{i}$ can be determined as:

$$
\frac{\partial J}{\partial \mathbf{Q}_{i}}=\frac{1}{6 V_{0}}(\mathbf{b} \wedge \mathbf{c})^{T}
$$

Here, $\mathbf{b}=\mathbf{Q}_{j}-\mathbf{Q}_{l}$ and $\mathbf{c}=\mathbf{Q}_{k}-\mathbf{Q}_{l}$ are edge vectors of the triangle opposing $\mathbf{Q}_{i}$. Thus, indices $j, k, l$ depend directly on $i$ and can be stored in a simple table. Using edge vector $\mathbf{c}$, the second derivative of $J$ is:

$$
\frac{\partial^{2} J}{\partial \mathbf{Q}_{j} \partial \mathbf{Q}_{i}}=\frac{1-\delta_{i j}}{6 V_{0}}\left[\begin{array}{ccc}
0 & -c_{z} & c_{y} \\
c_{z} & 0 & -c_{x} \\
-c_{y} & c_{x} & 0
\end{array}\right]
$$

Here, $\delta_{i j}$ is the Kronecker delta and matrix elements $c$ are components of the edge opposing points $\mathbf{Q}_{i}$ and $\mathbf{Q}_{j}$.

\subsection{Derivations for isotropic ground substance}

Following Weiss et al. [6], we use a Neo-Hookean material with shear modulus $\mu$ to model the response of the isotropic ground substance matrix:

$$
F_{G}=\frac{\mu}{2}\left(J^{-\frac{2}{3}} \operatorname{tr} \mathbf{C}-3\right)
$$

Stress tensor The individual terms required for the first derivative are:

$$
\frac{\partial J^{-\frac{2}{3}}}{\partial \mathbf{Q}_{i}}=-\frac{1}{9 V_{0}} J^{-\frac{5}{3}}(\mathbf{b} \wedge \mathbf{c})^{T} \quad, \quad \frac{\partial \operatorname{tr} \mathbf{C}}{\partial \mathbf{Q}_{i}}=2 \sum_{n=1}^{4}\left(\mathbf{D}_{n} \cdot \mathbf{D}_{i}\right) \mathbf{Q}_{n}^{T}
$$

The first derivative can then be calculated by:

$$
\frac{\partial F_{G}}{\partial \mathbf{Q}_{i}}=\frac{\mu}{2}\left(J^{-\frac{2}{3}} \frac{\partial \operatorname{tr} \mathbf{C}}{\partial \mathbf{Q}_{i}}+\frac{\partial J^{-\frac{2}{3}}}{\partial \mathbf{Q}_{i}} \operatorname{tr} \mathbf{C}\right)
$$


Elasticity tensor Again, we start with the individual terms of $F_{G}$ :

$$
\frac{\partial^{2} J^{-\frac{2}{3}}}{\partial \mathbf{Q}_{j} \partial \mathbf{Q}_{i}}=\frac{10}{9} J^{-\frac{8}{3}}\left(\frac{\partial J}{\partial \mathbf{Q}_{j}}\right)^{T} \frac{\partial J}{\partial \mathbf{Q}_{i}}-\frac{2}{3} J^{-\frac{5}{3}} \frac{\partial^{2} J}{\partial \mathbf{Q}_{j} \partial \mathbf{Q}_{i}}, \quad \frac{\partial^{2} \operatorname{tr} \mathbf{C}}{\partial \mathbf{Q}_{j} \partial \mathbf{Q}_{i}}=2\left(\mathbf{D}_{i} \cdot \mathbf{D}_{j}\right) \mathbf{1}
$$

Using these results, the second derivative is:

$$
\frac{\partial^{2} F_{G}}{\partial \mathbf{Q}_{j} \partial \mathbf{Q}_{i}}=\frac{\mu}{2}\left(\frac{\partial^{2} J^{-\frac{2}{3}}}{\partial \mathbf{Q}_{j} \partial \mathbf{Q}_{i}} \operatorname{tr} \mathbf{C}+\left(\frac{\partial \operatorname{tr} \mathbf{C}}{\partial \mathbf{Q}_{j}}\right)^{T} \frac{\partial J^{-\frac{2}{3}}}{\partial \mathbf{Q}_{i}}+\left(\frac{\partial J^{-\frac{2}{3}}}{\partial \mathbf{Q}_{j}}\right)^{T} \frac{\partial \operatorname{tr} \mathbf{C}}{\partial \mathbf{Q}_{i}}+J^{-\frac{2}{3}} \frac{\partial^{2} \operatorname{tr} \mathbf{C}}{\partial \mathbf{Q}_{j} \partial \mathbf{Q}_{i}}\right)
$$

\subsection{Derivations for collagen fiber family}

$F_{C}$ is a scalar function governing the non-linear behavior of the material along the main fiber orientation. We employ the formulation by Weiss et al. [6] and explicitly model the toe region of the stress-strain curve, which represents the successive recruitment of collagen fibers in ligaments under lower strains $\lambda<\lambda^{*}$.

$$
\begin{array}{lll}
\frac{\partial F_{C}}{\partial \lambda}=0 & , & \lambda<1 \\
\frac{\partial F_{C}}{\partial \lambda}=C_{3}\left(\exp \left(C_{4}(\lambda-1)\right)-1\right) & , & \lambda<\lambda^{*} \\
\frac{\partial F_{C}}{\partial \lambda}=C_{5} \lambda+C_{6} & , & \lambda \geq \lambda^{*}
\end{array}
$$

Stress tensor First, we note that:

$$
\frac{\partial F_{C}}{\partial \mathbf{Q}_{i}}=\frac{\partial F_{C}}{\partial \lambda} \frac{\partial \lambda}{\partial I_{4}} \frac{\partial I_{4}}{\partial \mathbf{Q}_{i}}=\frac{\partial F_{C}}{\partial \lambda} \frac{1}{2 \lambda} \frac{\partial I_{4}}{\partial \mathbf{Q}_{i}}
$$

This leaves us with finding the derivative for $I_{4}$. As we can use the result for $\partial J^{-\frac{2}{3}} / \partial \mathbf{Q}_{i}$ from (10), we continue with:

$$
\frac{\partial \mathbf{a C a}}{\partial \mathbf{Q}_{i}}=2\left(\mathbf{a} \cdot \mathbf{D}_{i}\right) \sum_{n=1}^{4}\left(\mathbf{a} \cdot \mathbf{D}_{n}\right) \mathbf{Q}_{n}^{T}
$$

We now know all the individual terms required for the derivative of $I_{4}$ :

$$
\frac{\partial I_{4}}{\partial \mathbf{Q}_{i}}=J^{-2 / 3} \frac{\partial \mathbf{a} \mathbf{C a}}{\partial \mathbf{Q}_{i}}+\frac{\partial J^{-\frac{2}{3}}}{\partial \mathbf{Q}_{i}} \mathbf{a C a}
$$

Using this in (15) delivers the stress tensor for $F_{C}$. 
Elasticity tensor The second derivatives of the stress-strain curve are:

$$
\begin{array}{lll}
\frac{\partial^{2} F_{C}}{\partial \lambda^{2}}=0 & , \lambda<1 \\
\frac{\partial^{2} F_{C}}{\partial \lambda^{2}}=C_{3} C_{4} \exp \left(C_{4}(\lambda-1)\right) & , \quad \lambda<\lambda^{*} \\
\frac{\partial^{2} F_{C}}{\partial \lambda^{2}}=C_{5} & , \lambda \geq \lambda^{*}
\end{array}
$$

We continue from (15), which gives us:

$$
\frac{\partial^{2} F_{C}}{\partial \mathbf{Q}_{j} \partial \mathbf{Q}_{i}}=\left(\frac{\partial^{2} F_{C}}{\partial \lambda^{2}}\left(\frac{\partial \lambda}{\partial I_{4}}\right)^{2}+\frac{\partial F_{C}}{\partial \lambda} \frac{\partial^{2} \lambda}{\partial I_{4}^{2}}\right)\left(\frac{\partial I_{4}}{\partial \mathbf{Q}_{j}}\right)^{T} \frac{\partial I_{4}}{\partial \mathbf{Q}_{i}}+\frac{\partial F_{C}}{\partial \lambda} \frac{\partial \lambda}{\partial I_{4}} \frac{\partial^{2} I_{4}}{\partial \mathbf{Q}_{j} \partial \mathbf{Q}_{i}}
$$

The second derivative of $\lambda$ w.r.t. $I_{4}$ is simply $\frac{\partial^{2} \lambda}{\partial I_{4}^{2}}=-\frac{1}{4{\sqrt{I_{4}}}^{3}}$. Next, we require the second derivative of $I_{4}$ :

$\frac{\partial^{2} I_{4}}{\partial \mathbf{Q}_{j} \partial \mathbf{Q}_{i}}=\frac{\partial^{2} J^{-\frac{2}{3}}}{\partial \mathbf{Q}_{j} \partial \mathbf{Q}_{i}} \mathbf{a} \mathbf{C a}+\left(\frac{\partial \mathbf{a C a}}{\partial \mathbf{Q}_{j}}\right)^{T} \frac{\partial J^{-\frac{2}{3}}}{\partial \mathbf{Q}_{i}}+\left(\frac{\partial J^{-\frac{2}{3}}}{\partial \mathbf{Q}_{j}}\right)^{T} \frac{\partial \mathbf{a} \mathbf{C a}}{\partial \mathbf{Q}_{i}}+J^{-\frac{2}{3}} \frac{\partial^{2} \mathbf{a C a}}{\partial \mathbf{Q}_{j} \partial \mathbf{Q}_{i}}$

The only new term in this equation is the second derivative of $\mathbf{a C a}$, which is:

$$
\frac{\partial^{2} \mathbf{a} \mathbf{C a}}{\partial \mathbf{Q}_{j} \partial \mathbf{Q}_{i}}=2\left(\mathbf{a} \cdot \mathbf{D}_{i}\right)\left(\mathbf{a} \cdot \mathbf{D}_{j}\right) \mathbf{1}
$$

Now all terms required for (19) are known and we can calculate the elasticity tensor for $F_{C}$.

\subsection{Derivations for volume conservation}

The penalty term controlling response of an element to volume changes is the same as for a nearly incompressible Neo-Hookean model [14]:

$$
F_{V}=\frac{K}{2}(J-1)^{2}
$$

with $K$ as the bulk modulus of the material.

Stress tensor The derivation of the first derivative is straight-forward:

$$
\frac{\partial F_{V}}{\partial \mathbf{Q}_{i}}=K(J-1) \frac{\partial J}{\partial \mathbf{Q}_{i}}
$$

Elasticity tensor The second derivative of the penalty term is:

$$
\frac{\partial^{2} F_{V}}{\partial \mathbf{Q}_{j} \partial \mathbf{Q}_{i}}=K\left(\left(\frac{\partial J}{\partial \mathbf{Q}_{j}}\right)^{T} \frac{\partial J}{\partial \mathbf{Q}_{i}}+(J-1) \frac{\partial^{2} J}{\partial \mathbf{Q}_{j} \partial \mathbf{Q}_{i}}\right)
$$




\section{$2.7 \quad$ In-situ stress}

When joints as the knee are in neutral position, the involved ligaments are usually still under tension [15]. Since this tension stabilizes the joint even when no muscles are active, the so-called in-situ stress plays an important role in joint kinematics. To achieve realistic simulation results, the employed FE model should thus include pre-stressed materials. In our model, we incorporate in-situ stress by increasing the magnitude of direction vectors in the fiber orientation field. By default, fiber orientation $\mathbf{a}$ is represented by a unit vector. If we use a modified orientation vector $\mathbf{a}^{\prime}=(1+c) \mathbf{a}$, the material is under a strain of $c$ even for an identity deformation gradient tensor.

\section{Estimating subject-specifc fiber orientations}

We assume that the subject-specific geometry of the ligament to be simulated is given by a high-resolution mesh. The attachment areas to the two bones the ligament connects are specified by two sets of vertices of this mesh, $A_{1}$ and $A_{2}$. The first step in our approach consists of finding the centerline between these two areas. This line provides a solid estimate of the main fiber orientation.

\subsection{Centerline extraction}

Centerline extraction is a common problem in medical image analysis, employed e.g. for vessel tracking or for generating fly-through paths for virtual endoscopy. For a brief overview of existing approaches see Bouix et al. [16]. As we can calculate the line start and end positions as the average positions of $A_{1}$ and $A_{2}$, we opt for a method based on minimal cost paths. Among all possible paths $P(s)$ connecting both points, we search the one that minimizes a specific metric $\mathcal{C}$. We include two components in $\mathcal{C}$ : first, the path should be as short as possible, and second, it should run at maximum distance from the mesh surface (i.e. as close to the medial sheet of the shape as possible). Both components are weighted by $\alpha \in[0,1]$ such that $\alpha=0$ results in the shortest possible path (a straight line) and $\alpha=1$ in a path around the geometric center. This leads to the following metric:

$$
\mathcal{C}(P)=\int_{0}^{1}\left|\frac{\partial P}{\partial s}\right|\left(\frac{\alpha}{d^{2}(P(s))}+(1-\alpha)\right) d s
$$

where $d(x)$ is the closest distance to the mesh surface from point $x$.

The search for the minimal cost path is set in a discrete setting. First, the surface mesh is rendered to an image volume and the corresponding distance transformation is calculated. Then, a graph is constructed with all object voxels as nodes and the 26-neighborhood for each voxel as edges. A Dijkstra search finally delivers the minimum cost path that serves as centerline for the ligament. 


\subsection{Local fiber orientation}

To calculate fiber orientations for all positions inside the segmented ligament mesh, we use the gradient of a diffusion field between centerline and mesh boundary. The diffusion field $D$ is constructed such that all voxels on the centerline have a value of 0 and all voxels on the mesh boundary a value of 1 . Generation of this field is a classical Dirichlet problem. In our current implementation we use the following geometric method: for each point $x$ within the mesh, the closest point on the centerline $C(x)$ is determined. A ray is cast from $C(x)$ through $x$ until it hits the mesh boundary at $B(x)$. We then set $D(x)=|x-C(x)| /|B(x)-C(x)|$.

We assume that fibers are oriented parallel to the boundary for positions on the boundary and parallel to the centerline for positions on the centerline. In between, fiber directions are interpolated so that they run perpendicular to the gradient $\nabla D$, i.e. on an isosurface of the diffusion field. To determine the orientation vector $\mathbf{a}(x)$, the direction of the centerline at $C(x)$ is projected onto the isosurface at $x$ and normalized. Finally, orientation vectors for all $x$ inside a specific tetrahedral element are averaged to yield a for this element.

\section{Material for subject-specific ligament model}

As proof of concept for the presented methods, we simulate the medial collateral ligament (MCL) in a subject-specific knee joint.

\subsection{Image data}

MRI data was used as source for the geometry of the simulation. An institutional medical-ethics committee approved the image acquisition and the subject gave his written consent. The employed dataset covers the right knee of a healthy male subject (age: 38 ) in full extension. It was acquired using a $1.5 \mathrm{~T}$ Siemens MRI device with Flair Sagittal 3D T1 protocol and $256 \times 256$ matrix. Image resolution was $0.5 \times 0.5 \mathrm{~mm}$ with a slice thickness of $1.0 \mathrm{~mm}$.

\subsection{Rheological parameters}

While it is possible to extract subject-specific geometry for a living subject, we can think of no way to determine subject-specific material properties. For our simulation of the MCL, we thus employ the rheological parameters presented for this structure by Weiss et al. [6]: $\mu=27.7 \mathrm{MPa}, \lambda^{*}=1.03, C_{3}=2.07 \mathrm{MPa}$, $C_{4}=61.44, C_{5}=640.7 \mathrm{MPa}$. In-situ strain was set to $2.9 \%$. To achieve near incompressible behavior, the bulk modulus was set to $K=1000$.

\section{$5 \quad$ Experiments and results}

\subsection{Verification}

To verify our implementation of the material model, we conducted a number of tests on a synthetic cube shape of $1 \mathrm{~mm}$ edge length. The employed mesh 

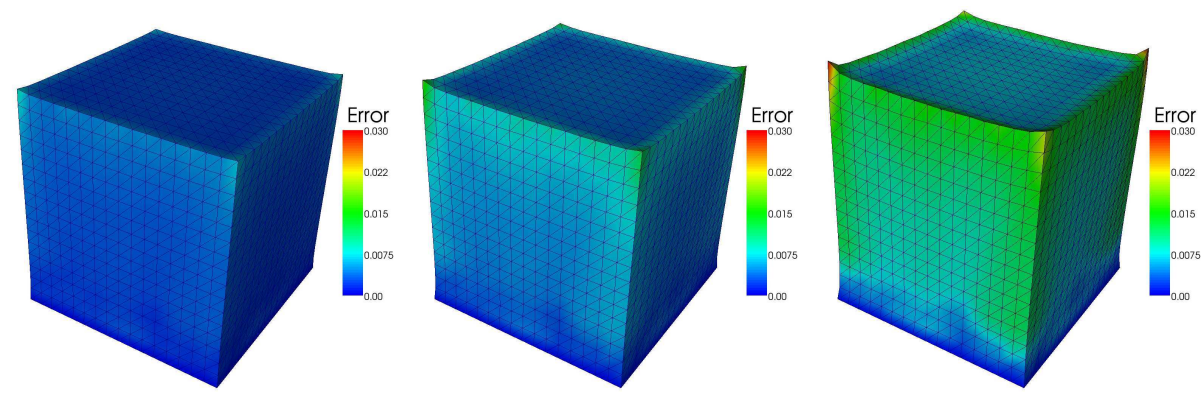

Fig. 1. Node positioning errors to FEBio reference solution for tensile loading, colorcoded from 0 to $3 \%$. From left to right, applied tensile forces per node are $0.05 \mathrm{~N}, 0.1 \mathrm{~N}$, and $0.2 \mathrm{~N}$, respectively. Fibers are oriented parallel to forces.
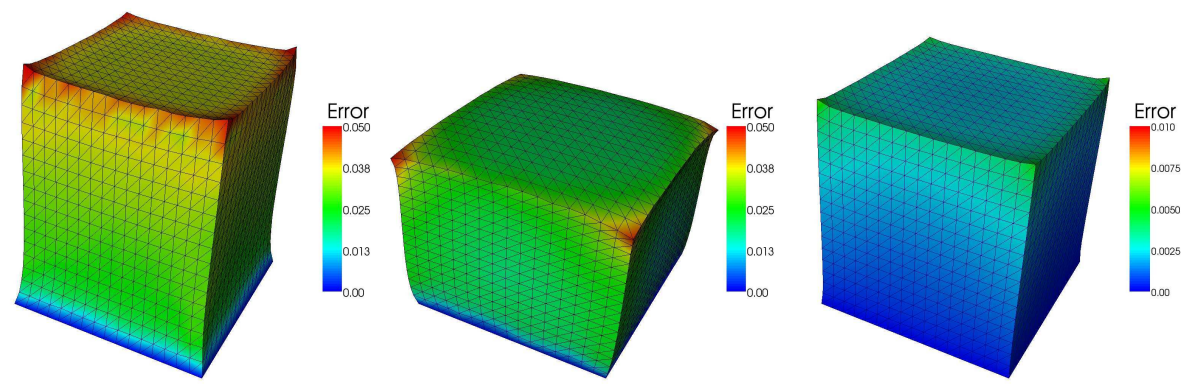

Fig. 2. Node positioning errors for alternative test cases. Left: Tensile forces of $0.05 \mathrm{~N} /$ node, but with fibers oriented perpendicular to forces. Center: Compressing forces of $0.05 \mathrm{~N} /$ node. Right: Same scenario as in Fig. 1 center, but compared to FEBio without Augmented Langrangian. Note the different error scales (up to 6\% for left and center, up to $1 \%$ for right).

consisted of 4316 nodes and 20737 tetrahedral elements. Material parameters were set to the values given in Sec. 4.2. Constraints were used to fix positions of all nodes on the lower side of the cube. To all nodes on the upper side of the cube, we applied constant forces pointing upwards (tensile strain) or downwards (compressive strain). An implicit solver with velocity damping was run until convergence and the resulting deformed mesh was exported.

The scenario was then simulated with the FEBio software, using the same mesh and identical boundary conditions. The material model in FEBio was set to transversly isotropic Mooney-Rivlin with the same parameters as above. Volume conservation was enforced using the Augmented Lagrangian approach. The solution was obtained for the quasi-static case with the default BFGS solver.

We compared our results to the FEBio reference solution for a variety of cases. All positioning errors are expressed in percent of the edge length of the cube in 
Table 1. Results of experiments on the synthetic cube with fibers oriented parallel to tensile forces. For each load, the resulting average fiber strain and volume change is given. From comparison to the FEBio solution, we determined the average positioning error for all nodes on the top surface of the cube as well as the maximum error.

\begin{tabular}{rrrrrr}
\hline Load/node & Total load & Avg. fiber strain & Vol. change & Avg. error & Max. error \\
\hline $0.02 \mathrm{~N}$ & $5.8 \mathrm{~N}$ & $+1.6 \%$ & $+0.2 \%$ & $0.1 \%$ & $0.4 \%$ \\
$0.05 \mathrm{~N}$ & $14.5 \mathrm{~N}$ & $+3.0 \%$ & $+0.5 \%$ & $0.3 \%$ & $1.0 \%$ \\
$0.10 \mathrm{~N}$ & $28.9 \mathrm{~N}$ & $+4.9 \%$ & $+1.1 \%$ & $0.5 \%$ & $1.9 \%$ \\
$0.20 \mathrm{~N}$ & $57.8 \mathrm{~N}$ & $+8.8 \%$ & $+2.2 \%$ & $0.9 \%$ & $3.4 \%$ \\
\hline
\end{tabular}

Table 2. Additional experiments on the synthetic cube. The first two cases represent unusual load scenarios, the last two (marked with *) are compared to the FEBio solution without Augmented Lagrangian.

\begin{tabular}{rrrr}
\hline & Set-up & Vol. change Avg. error Max. error \\
\hline Fibers perpendicular to tensile load $(0.05 \mathrm{~N})$ & $+0.7 \%$ & $3.9 \%$ & $5.8 \%$ \\
Fibers parallel to compressive load $(0.05 \mathrm{~N})$ & $-0.5 \%$ & $2.4 \%$ & $5.6 \%$ \\
Fibers parallel to compressive load $(0.10 \mathrm{~N}){ }^{*}$ & $+1.1 \%$ & $0.3 \%$ & $0.6 \%$ \\
Fibers perpendicular to tensile load $(0.05 \mathrm{~N}){ }^{*}$ & $+0.7 \%$ & $0.4 \%$ & $0.5 \%$ \\
\hline
\end{tabular}

rest configuration. Table 1 lists results for tensile forces typically encountered in ligaments under physiological conditions. In Fig. 1, the corresponding node positioning errors are visualized. Table 2 lists results for cases that usually do not occur in ligaments (as loading perpendicular to fiber orientation) and also compares our results to the FEBio solution without Augmented Lagrangian. Figure 2 again visualizes the corresponding node positioning errors.

\subsection{MCL simulation}

Geometry of femur, tibia and MCL was segmented manually from the MRI dataset described in Sec. 4. For the centerline extraction, we set $\alpha=0.1$. Figure 3 shows the segmented ligament mesh and the resulting centerline. From the surface, we generated a volumetric mesh consisting of 1193 nodes and 5911 tetrahedra. Using the presented rheological parameters for the ligament and rigid bones, a dynamic simulation was set up with the SOFA software [17]. An Euler implicit scheme was used for time integration, and a modified conjugate gradient solver [18] was employed to solve the system of equations. While the tibia was fixed, the femur was moved manually to different flexion angles. Figure 4 shows the resulting strain energy within the MCL for these cases. On an Intel Core 2 Duo notebook with $2.2 \mathrm{GHz}$ CPU the obtained framerate was 15 frames/second (including simulation and visualization). 


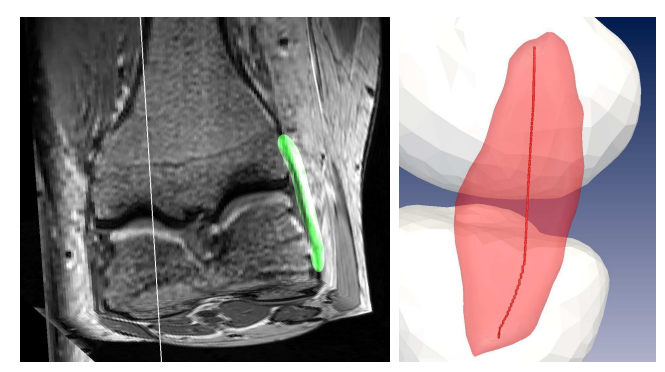

Fig. 3. Segmented ligament mesh together with the original MRI data (left) and the extracted centerline connecting the attachment areas of femur and tibia (right).
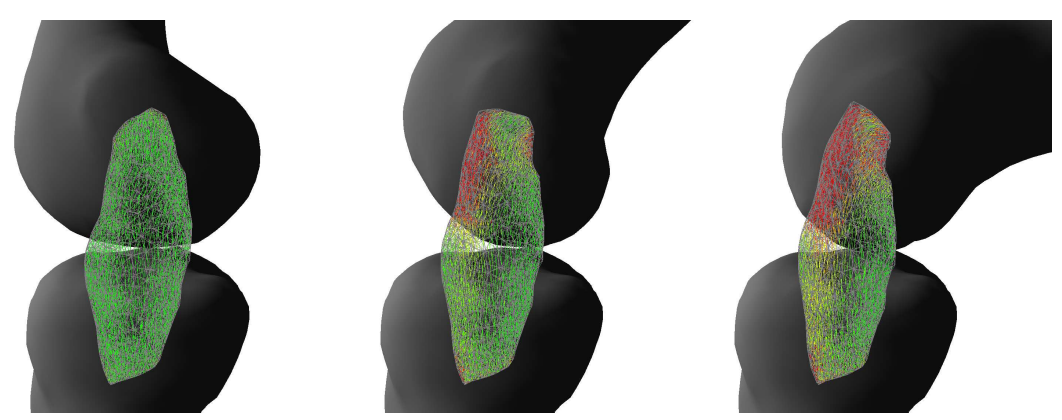

Fig. 4. Simulation of strain in the MCL at 0,45 , and 90 degrees of knee flexion. Strain energy is color-coded from green to red, the lines indicate local fiber orientation.

\section{Discussion}

The comparison of our FE model to FEBio results in average node positioning errors $<1 \%$ for the intended application, which we regard as sufficient accuracy. As a comparison to FEBio without Augmented Lagrangian reveals, the discrepancies are mainly due to the different approach to volume conservation. Our method to estimate fiber orientations requires only minimal interaction for the definition of attachment areas (which are required in any case) and delivers mostly convincing results. However, some problems occur at the attachment areas, where orientation cannot be determined reliably when the centerline does not start at the mesh boundary. While the obtained framerate is not yet suitable for real-time simulation, it is nonetheless much faster than classic FE solvers. Indeed, the largest challenge we see for future virtual surgeries is not the performance of the FE model, but the generation of accurate subject-specific geometries: ligaments are hardly visible in standard MRI data, and small variations in the segmentation can affect simulation results considerably. As a next step, we plan to validate the presented methods against experimental studies on real ligament tissue. 
Acknowledgment This work is supported by the EU Marie Curie project 3D Anatomical Human (MRTN-CT-2006-035763).

\section{References}

1. Woo, S.L.Y., Debski, R.E., Withrow, J.D., Janaushek, M.A.: Biomechanics of knee ligaments. Am J Sports Med 27(4) (1999) 533-543

2. Chen, J.X., Wechsler, H., Pullen, J.M., Zhu, Y., MacMahon, E.B.: Knee surgery assistance: patient model construction, motion simulation, and biomechanical visualization. IEEE Trans Biomed Eng 48(9) (2001) 1042-1052

3. Ramaniraka, N.A., Saunier, P., Siegrist, O., Pioletti, D.P.: Biomechanical evaluation of intra-articular and extra-articular procedures in anterior cruciate ligament reconstruction: a finite element analysis. Clinical Biomechanics 22 (2007) 336-343

4. Ellis, B.J., Lujan, T.J., Dalton, M.S., Weiss, J.A.: Medial collateral ligament insertion site and contact forces in the ACL-deficient knee. J Orthop Res 24(4) (2006) $800-810$

5. Fernandez, J.W., Hunter, P.J.: An anatomically based patient-specific finite element model of patella articulation: towards a diagnostic tool. Biomech Model Mechanobiol 4 (2005) 20-38

6. Weiss, J.A., Maker, B.N., Govindjee, S.: Finite element implementation of incompressible, transversely isotropic hyperelasticity. Comput. Methods Appl. Mech. Engrg. 135 (1996) 107-128

7. Pioletti, D.P., Rakotomanana, L.R., Benvenuti, J.F., Leyvraz, P.F.: Viscoelastic constitutive law in large deformations: application to human knee ligaments and tendons. J Biomech 31 (1998) 753-757

8. Wu, W., Heng, P.A.: An improved scheme of an interactive finite element model for 3D soft-tissue cutting and deformation. Visual Comput 21 (2005) 707-716

9. Picinbono, G., Delingette, H., Ayache, N.: Non-linear anisotropic elasticity for real-time surgery simulation. Graphical Models 65 (2003) 305-321

10. Teran, J., Sifakis, E., Blemker, S.S., Ng-Thow-Hing, V., Lau, C., Fedkiw, R.: Creating and simulating skeletal muscle from the visible human data set. IEEE Trans Visualization and Computer Graphics 11(3) (2005) 317-328

11. Joldes, G.R., Wittek, A., Miller, K.: Suite of finite element algorithms for accurate computation of soft tissue deformation for surgical simulation. Med Image Anal In press (2009)

12. Taylor, Z.A., Comas, O., Cheng, M., Passenger, J., Hawkes, D.J., Atkinson, D., Ourselin, S.: On modelling of anisotropic viscoelasticity for soft tissue simulation: Numerical solution and GPU execution. Med Image Anal 13(2) (2009) 234-244

13. Delingette, H.: Triangular springs for modeling nonlinear membranes. IEEE Trans Vis Comp Graph 14(2) (2008) 329-341

14. Bower, A.F.: Applied Mechanics of Solids. CRC (2009)

15. Weiss, J.A., Gardiner, J.C.: Computational modeling of ligament mechanics. Critical Reviews in Biomedical Engineering 29(4) (2001) 1-70

16. Bouix, S., Siddiqi, K., Tannenbaum, A.: Flux driven automatic centerline extraction. Med Image Anal 9 (2005) 205-221

17. Allard, J., Cotin, S., Faure, F., Bensoussan, P.J., Poyer, F., Duriez, C., Delingette, H., Grisoni, L.: SOFA - an open source framework for medical simulation. In: Medicine Meets Virtual Reality (MMVR'15), Long Beach, USA (2007)

18. Baraff, D., Witkin, A.P.: Large steps in cloth simulation. In: Proc SIGGRAPH. (1998) 43-54 